\title{
Importancia de la categorización territorial para la descentralización y las relaciones intergubernamentales en Colombia**
}

\section{Importance of territorial categorization for decentralization and intergovernmental relations in Colombia}

SUMARIO

Introducción. 1. Fundamentos teóricos y conceptuales del diseño territorial descentralizado. 2. Limitaciones a la descentralización y la autonomía territorial presentes en el diseño normativo. 2.1. Marco normativo de la categorización municipal. 2.2. Marco normativo de la categorización departamental. 3. Alternativas de la categorización municipal. 3.1. Criterios de categorización municipal. 3.2. Factores de diferenciación del régimen municipal. 4. Alternativas de la categorización departamental. 5. Diseño municipal y categorización a nivel internacional. 5.1. Principio de subsidiariedad. 5.2. Esquemas de asociatividad territorial. 5.3. Categorización municipal. Conclusiones. Referencias.

\footnotetext{
* Investigadora principal del proyecto, profesora investigadora de planta de tiempo completo del Programa de Administración Pública de la Institución Universitaria Politécnico Grancolombiano. Administradora pública y magíster en Administración Pública de la ESAP. Estudiante del doctorado en Modelado en Política y Gestión Pública de la Universidad Jorge Tadeo Lozano (Bogotá, Colombia).Contacto: naduquec@poligran.edu.co

*** Este artículo se desarrolló en el marco de dos proyectos de investigación. El primero, "Categorización municipal en Colombia", es el proyecto de grado para el Programa de Doctorado en Modelado en Política y Gestión Pública de la Universidad Jorge Tadeo Lozano que cursa actualmente la autora. El segundo, "Capacidad institucional de los municipios pequeños en Colombia", desarrollado y financiado, igual que el primero, en su totalidad por la Institución Universitaria Politécnico Grancolombiano, Bogotá (Colombia). Ambos proyectos pertenecen al área de Administración Pública del grupo de investigación Economía, Derechos y Globalización de la IUPG.

Recibido el 26 de septiembre de 2016, aprobado el 1. ${ }^{\circ}$ de marzo de 2017.

Para citar el artículo: DuQue CANTE, N. Importancia de la categorización territorial para la descentralización y las relaciones intergubernamentales en Colombia. Derecho del Estado n. ${ }^{\circ}$ 38, Universidad Externado de Colombia, enero-junio de 2017, pp. 67-95. DOI: https://doi.org/10.18601/01229893.n38.03
} 
RESUMEN

Este artículo reflexiona sobre la importancia de la categorización territorial, en especial de los municipios, para el mejoramiento de la capacidad de respuesta del Estado en la provisión de bienes y servicios sociales. La categorización municipal debería derivar a su vez en un replanteamiento del papel del departamento y en el reconocimiento de las diferencias que manifiestan estas entidades en la actualidad.

Con el análisis realizado se evidenció que la prestación directa está sujeta, en la mayoría de los países de América Latina y Europa, igual que en Colombia, a un diseño intergubernamental en múltiples dimensiones, que incluyen concurrencia, coordinación, complementariedad y subsidiariedad, entre los tres niveles y esquemas asociativos territoriales. Sin embargo, aunque este diseño reconoce las diferencias entre los tres niveles del Estado, no reconoce aquellas que están presentes al interior de los dos niveles territoriales existentes en nuestro país, lo cual debería resolverse a través del establecimiento de categorías municipales y departamentales.

PALABRAS CLAVE

Categorización territorial, municipios, departamentos, descentralización, relaciones intergubernamentales.

\section{ABSTRACT}

This article reflects on the importance of the territorial categorization, especially of the municipalities, for the improvement of the capacity of the State to respond in terms of the provision of social goods and services. The municipal categorization should, in turn, lead to a rethinking, not only of the department's role, but also of the recognition of the differences that these entities manifest today.

The analysis showed that direct provision is subject, in most countries of Latin America and Europe, as in Colombia, to an intergovernmental design in multiple dimensions, including concurrence, coordination, complementarity and subsidiarity, among The three levels and territorial associative schemes. However, although this design recognizes the differences between the three levels of the State does not recognize those that are present within the two territorial levels existing in our country, which should be resolved through the establishment of municipal and departmental categories. 
KEYWORDS

Territorial categorization, municipalities, departments, decentralization, intergovernmental relationship

\section{INTRODUCCIÓN}

Uno de los desarrollos más importantes para la organización del Estado en Colombia ha sido la descentralización territorial. Como novedad, planteó el reto de redefinir los vínculos entre el nivel nacional y las entidades territoriales, en aras de procurar la reivindicación de los territorios como factor trascendental dentro de la lógica de modernización administrativa y mejoramiento social de las poblaciones.

Pero, si bien la descentralización territorial prometía generar cambios revolucionarios, no solo en el diseño normativo sino, principalmente, en la puesta en marcha de sus preceptos, los resultados no han sido los que se proyectaron en sus orígenes, tanto en lo relativo al funcionamiento de las entidades territoriales como en lo que respecta al mejoramiento permanente de la calidad de vida. Asimismo, los resultados han hecho evidente que aunque estuvieran dadas las condiciones de capacidad institucional a nivel social y político para la descentralización, todavía estaría pendiente el adecuado diseño territorial del Estado.

Dicho diseño se refiere a la forma como se define el papel de los tres niveles del Estado, por un lado, en lo relativo a las funciones que le corresponde cumplir a cada uno de ellos, y por otro, al reconocimiento de las diferencias que existen entre los dos niveles territoriales de nuestro país, así como al interior de cada uno de ellos. De acuerdo con la Constitución de 1991, estas diferencias deberían conducir a un trato particular en materia de organización, funciones y autoridades para el caso de los municipios. De igual manera, aunque la Carta Política no considera a los departamentos, una categorización municipal debería obligar a una adaptación del departamento y por ende a una categorización del nivel intermedio.

A partir de una revisión normativa y documental se encontró que a pesar del mandato constitucional, y del desarrollo de leyes que establecen categorías para municipios, distritos y departamentos, en la actualidad esto no genera efectos en la configuración ni en el funcionamiento de las entidades territoriales, independientemente de sus potencialidades y/o limitaciones. En el caso del departamento, la situación es similar, ya que aunque sus complejidades lo hacen distinto al municipio, también está diseñado de manera homogénea y artificial, desconociendo, en todo caso, su naturaleza como ente intermedio, así como las particularidades que lo distinguen cuando se atiende a sus vínculos con el nivel local. Esto ha conducido a que cada uno de estos niveles actúe, por un lado, desarticulado de las particularidades de 
su territorio, y por el otro, al margen de la lógica intergubernamental ${ }^{1}$ de un Estado unitario.

Adicionalmente, y con el propósito de articular el análisis de nuestro país a la luz del contexto internacional, el documento avanza en la reflexión acerca de las posibilidades que ofrece la categorización en los términos establecidos por la Constitución de 1991, y en las diferentes alternativas de diseño intergubernamental presentes en varios países de América Latina y Europa y que buscan resolver las exigencias de la prestación directa de bienes y servicios por parte del Estado.

\section{FUNDAMENTOS TEÓRICOS Y CONCEPTUALES DEL DISEÑO TERRITORIAL DESCENTRALIZADO}

La descentralización territorial de nuestro país tiene su origen en la transformación del Estado de la década de los años ochenta del siglo xx, que tuvo lugar en toda la región latinoamericana. Esta transformación, que condujo de un Estado basado en el modelo "desarrollista" de la CEPAL a un Estado basado en la doctrina neoliberal, se caracterizó en un primer momento por el reconocimiento e intervención en varios tipos de crisis que afectaron al Estado y su Administración Pública. Por un lado estaba la crisis de la deuda, que constituyó la principal justificación para la reforma del Estado con énfasis neoliberal y su propuesta de orientación hacia un Estado mínimo. De igual manera, se estaba ante la crisis del modelo de sustitución de importaciones, cuya lógica transgredía las nacientes y arrasadoras tendencias hacia la globalización y sus dinámicas de libre flujo de mercancías y capitales. Finalmente, se asistía a la crisis del centralismo, derivada de una larga tradición de organización administrativa cuya manifestación principal fue un proceso de toma de decisiones que excluía las particularidades territoriales y en especial las del nivel local (Restrepo, 2004; Finot, 2007; Borja, 1987). Todos estos eventos, especialmente el último, condujeron a una crisis de gobernabilidad del Estado, debido a los fuertes cuestionamientos que se plantearon frente a su pertinencia y funcionalidad como expresión de los intereses sociales y económicos.

Por otro lado, la primera y la tercera de estas crisis, si bien tienen puntos de encuentro, son distintas manifestaciones de la crisis del Estado. En el primer caso, la crisis evidenció que el Estado necesitaba cada vez mayores recursos para cumplir su creciente número de obligaciones, conduciéndolo a un desequilibrio fiscal cada vez menos manejable. A su vez, esto coincidió con que el mayor gasto público no derivó en mayores niveles de cobertura y

1 Las relaciones intergubernamentales (RIG) se pueden definir como los vínculos de coordinación, cooperación, apoyo mutuo e intercambio de acciones que se dan entre dos o más instancias de gobierno (Díaz, 2006). 
calidad de los bienes y servicios sociales, lo cual condujo a la consideración de que el proceso de toma de decisiones de manera centralizada impedía la adecuada focalización de los recursos públicos con miras a atender las necesidades de las comunidades hacia las cuales iban dirigidos. Es decir que, aunque pudo haberse dado un proceso de excesivo endeudamiento con unos adecuados niveles de satisfacción de las necesidades sociales, en la práctica se evidenció una combinación de gasto excesivo, con recursos provenientes de la deuda, junto con niveles negativos de impacto social en la asignación de los recursos (Huerta, 2005).

Todos estos fenómenos derivaron en una redefinición del Estado cuya orientación buscó ajustar su funcionamiento en procura de responder a las diferentes presiones del contexto. Moncayo (2006) ${ }^{2}$ ilustra de forma clara cómo la transformación condujo a un vaciamiento del poder del Estado en tres direcciones distintas. En primer lugar, hacia arriba, por efectos de la globalización y la supranacionalización, cuyo lugar de llegada han sido las instituciones internacionales y supranacionales; en segundo lugar, horizontalmente, por el desmonte del Estado de Bienestar, la privatización y la desregulación, cuyo lugar de llegada ha sido el mercado; y en tercer lugar, hacia abajo, a las entidades subnacionales a través de fenómenos como la descentralización.

Esta diferenciación de las crisis permite a su vez explicar por qué, aunque la tendencia general de la transformación debería haber conducido a un proceso sistemático de Estado mínimo neoliberal, en la práctica el Estado se redujo en algunos lugares pero terminó aumentando su tamaño donde antes era moderado (Oszlak, 2000). El nivel nacional se redujo, por efectos de las privatizaciones masivas, y por el traslado de competencias hacia los niveles subnacionales de gobierno, pero este último fenómeno terminó haciendo que el tamaño del sector público creciera de manera importante en los territorios. En consecuencia, este incremento de tamaño condujo a un mayor gasto público, tanto por efectos del crecimiento de las burocracias como por el aumento de la demanda ciudadana por una mayor cantidad y calidad de bienes públicos en el nivel territorial (Gómez, 2010).

Por otro lado, al incorporarse la descentralización territorial como el mecanismo ideal para contrarrestar la concentración de poder en los niveles nacionales de los Estados latinoamericanos, se generaron distintas interpretaciones frente a las virtudes que dicha herramienta podía generar. La descentralización se consideró la solución más conveniente para enfrentar la crisis de gobernabilidad, debido a la baja capacidad de respuesta del Estado frente a las necesidades sociales; las deficiencias de representación política derivadas de una dinámica política centenaria ubicada en el nivel

2 Sobre este punto también se puede consultar a RESTREPO (2004), quien hace referencia a los fenómenos de globalización, privatización y descentralización mencionados por Moncayo. 
nacional; la crisis del sistema político, cuya principal manifestación fue la pérdida de legitimidad que amenazaba la estabilidad del régimen, y la crisis administrativa evidenciada por la ineficiencia e ineficacia del gasto público. Adicionalmente, y debido a varios de los aspectos anteriores, se consideró que la descentralización era la mejor alternativa para consolidar la democracia local (Gaitán y Moreno, 1992).

Más allá de los efectos en el imaginario social y político, la descentralización es en la práctica un fenómeno de carácter normativo que busca generar efectos en la forma como se distribuyen y administran los asuntos públicos entre el nivel nacional y las entidades territoriales. Tal como está planteado desde el federalismo fiscal, en el diseño territorial se debe "establecer una combinación óptima entre la centralización y la descentralización en la asignación de competencias entre los diferentes niveles de gobierno" (Díaz, 2002 , p. 5). Esta asignación debe ser coherente con las ventajas de cada uno de los niveles del Estado en relación con las posibilidades de lograr mayor eficiencia y equidad en la provisión de bienes y servicios públicos. En estos términos, el federalismo fiscal propende por una fórmula adecuada entre centralización y descentralización, en la que el nivel nacional debe reservarse aquellas funciones que superan los ámbitos regional y local, y los gobiernos subnacionales deben ocuparse de aquellos asuntos que se ubiquen en sus respectivas jurisdicciones territoriales.

Una distribución de competencias adecuada, que responda a una fórmula conveniente de asignación de funciones y recursos a nivel nacional y territorial, requiere, además, del cumplimiento de tres requisitos que Bird (2001) considera necesarios para que se produzca el "principio de correspondencia", que son:

1. que el área que recibe el beneficio cargue con los costos;

2. que la responsabilidad de gastar coincida con la de recaudar, y

3. que exista una correspondencia entre la responsabilidad de financiamiento y la responsabilidad política.

En estas condiciones, si una entidad territorial asume una competencia cuyo impacto se ubica dentro de su respectivo territorio se esperaría que la misma asuma la responsabilidad de los costos que origina. Esta situación se debe fundar a su vez en la condición previa que supone que la entidad territorial debe contar con la capacidad de recaudo suficiente para financiar las necesidades y problemáticas que se generan en su propia jurisdicción (Pinilla y otros, 2015). Finalmente, debe existir una relación entre las autoridades que toman las decisiones en materia de financiación de bienes y servicios públicos y la respectiva jurisdicción territorial.

La mayor descentralización, haciendo referencia a una adecuada distribución de competencias, lograría mayor eficiencia en la asignación de los recursos públicos en la medida que los beneficios no serían de aplicación universal sobre la población. Por el contrario, entre más homogéneas y pe- 
queñas sean las comunidades, más eficiente será la provisión de servicios, permitiendo responder de forma más cercana a las preferencias particulares. Para ello, los gobiernos tendrán la oportunidad de consultar las necesidades, problemáticas y preferencias de la población de forma más precisa. En estos términos, cuando se produce una mayor autonomía territorial derivada de la articulación entre ingresos y provisión de bienes y servicios públicos es más fácil que las personas relacionen los beneficios con el pago en impuesto que han realizado, lo cual derivaría en mayores niveles de recaudo (Subdere, 2009).

No obstante, aunque el principio de correspondencia supone un orden lógico, parte de la existencia de condiciones ideales que no se producen del todo en la realidad de los países, en particular los del Tercer Mundo. En primer lugar, la existencia de desequilibrios interregionales constituye un obstáculo para el proceso en la medida en que cualquier intento de profundización de la descentralización, tanto en materia de gastos como de impuestos, puede conducir a agravar tales desequilibrios. Sin embargo, si el propósito no es lograr mayores niveles de equidad interregional, entonces es recomendable que se mantenga la correspondencia entre gastos y recaudo (Porto, 2008). Incluso, para Wiesner (2002), si se quiere hablar de un verdadero proceso de descentralización el crecimiento de los ingresos tributarios locales debe ser superior al de los ingresos tributarios nacionales, lo cual no se refiere solo a un incremento de las transferencias sino principalmente a un incremento de los recursos propios. En caso contrario, la búsqueda de la igualación entre las regiones conduciría a justificar como conveniente y necesaria la existencia de transferencias intergubernamentales, y con ello a la mayor concentración de impuestos en el nivel nacional. Incluso, permitiría justificar la mayor injerencia del nivel nacional en los territorios, conduciendo a la implementación de políticas centralistas orientadas a reducir también los niveles de transferencias y otros recursos nacionales hacia las entidades territoriales (Ramírez, 2015).

El proceso de diseño descentralizado es, a su vez, la base para determinar los niveles de autonomía territorial y la forma como se desarrollan las relaciones intergubernamentales. De acuerdo con la Constitución de 1991, en Colombia las entidades territoriales tienen autonomía para la gestión de sus asuntos propios. Esta autonomía no es una autonomía legislativa, sino una autonomía para tomar decisiones al interior de su territorio sin la injerencia de autoridades externas (Duque, 2009). La toma de decisiones está sujeta a la capacidad que tenga la entidad para financiar dichas decisiones con sus propios recursos. Por ende, entre mayor dependencia tenga el ente territorial frente a los recursos provenientes de la nación para financiar las necesidades de su población, menor autonomía territorial le asiste. En estos términos, las relaciones intergubernamentales, entendidas como "los vínculos de coordinación, cooperación, apoyo mutuo e intercambio de acciones que se dan entre dos o más instancias de gobierno" (Díaz, 2006, p. 38), terminarían siendo relaciones de dependencia y coordinación para la ejecución de las políticas 
nacionales y no relaciones de articulación para la generación de proyectos conjuntos entre niveles del Estado. No obstante, se afectarían las relaciones verticales, y la debilidad fiscal de los territorios podría conducir al fotalecimiento de las relaciones de carácter horizontal, mediante la conformación de esquemas de asociatividad territorial.

\section{LIMITACIONES A LA DESCENTRALIZACIÓN Y LA AUTONOMÍA TERRITORIAL PRESENTES EN EL DISEÑO NORMATIVO}

En Colombia las entidades territoriales cuentan con funciones propias y autoridades para tomar las decisiones de su territorio, pero en la mayoría de los casos los recursos son insuficientes. Por ello, aunque la Constitución reconoce la autonomía territorial, no la garantiza, en especial porque no garantiza que los recursos coincidan con el nivel de responsabilidades asignadas, conduciendo a la existencia de autonomía a niveles demasiado bajos e insignificantes.

A las limitaciones introducidas por la rigidez del marco normativo territorial se suma el énfasis puesto en la gestión organizacional introducido por la Nueva Gestión Pública, y el que la escasez de los recursos ha convertido al municipio, en la mayoría de los casos, en una entidad meramente ejecutora de políticas y programas nacionales. En la práctica, las autoridades locales están más preocupadas por el cumplimiento de responsabilidades administrativas que por atender a sus ciudadanos. Los asuntos propios, que se derivan de la cotidianidad de la vida local, pasan a ocupar un segundo lugar frente a la avalancha de requerimientos burocráticos que deben cumplir las administraciones municipales en nuestro país frente al nivel nacional.

\subsection{Marco normativo de la categorización municipal}

En Colombia, los municipios pequeños difícilmente podrán superar los obstáculos a los que se enfrentan (Duque, 2010), pero, dado que el diseño normativo no reconoce esta realidad, se hace imposible un ejercicio real de la autonomía territorial, aunque sea limitada, para estas entidades territoriales. Para muchos de ellos, incluso, sería más conveniente reconocer la imposibilidad de concretar la autonomía territorial así sea a niveles mínimos, que seguirlos considerando equivocadamente entidades territoriales. De hecho su realidad se ajusta más a la de una unidad desconcentrada. En el caso de los demás municipios, sin embargo, la categorización permitiría ajustar las particularidades de la entidad con la definición de la misma en el ordenamiento normativo, mejorando las probabilidades de aprovechar sus potencialidades y ventajas.

Como posibilidad, la categorización municipal se encuentra definida en el artículo 320 la Constitución Política de 1991. Esta norma establece como 
criterios de categorización los factores de población, recursos fiscales, importancia económica y situación geográfica. Aunque esta directriz involucra un nivel importante de complejidad, la intención de proyectar el territorio sobre las normas no es novedosa en el ordenamiento institucional colombiano. Su origen se puede ubicar en la reforma constitucional de 1945 y más adelante con la reforma de 1968. En ambos actos legislativos se plantea la posibilidad de que la ley establezca diferentes categorías municipales tomando en cuenta la población, los recursos y la importancia económica. La diferencia es que con la Constitución de 1991 se incluye un nuevo criterio de categorización, la situación geográfica, y que los efectos de ubicar a los municipios en estas categorías deberían derivar en diferencias en tres aspectos específicos, la organización, el gobierno y la administración, y no solo en el último como estaba considerado con la Constitución anterior.

\begin{tabular}{|l|l|l|l|}
\hline \multicolumn{2}{|c|}{ CONSTITUCIÓN DE 1991 } & \multicolumn{2}{c|}{ CONSTITUCIÓN DE 1886 } \\
\hline $\begin{array}{c}\text { Criterio de } \\
\text { categorización } \\
\text { municipal }\end{array}$ & $\begin{array}{c}\text { Efectos de la } \\
\text { categorización }\end{array}$ & $\begin{array}{c}\text { Criterio de } \\
\text { categorización } \\
\text { municipal }\end{array}$ & $\begin{array}{c}\text { Efectos de la } \\
\text { categorización }\end{array}$ \\
\hline $\begin{array}{l}\text { - Población } \\
\text { - Recursos fiscales } \\
\text { - Importancia } \\
\text { económica } \\
\text { - Situación } \\
\text { geográfica }\end{array}$ & $\begin{array}{l}\text { - Organización } \\
\text { - Gobierno }\end{array}$ & $\begin{array}{l}\text { - Población } \\
\text { - Recursos Fiscales }\end{array}$ & - Admininistración \\
\hline
\end{tabular}

Fuente: elaboración propia.

\subsubsection{Contenidos de las leyes 617 de 2000 y 1551 de 2012}

En comparación con la Constitución Política de 1991, los desarrollos normativos han sido lánguidos y superficiales al reglamentar la categorización territorial. El interés por reconocer las diferentes dimensiones de la realidad territorial en categorías con amplio contenido cualitativo se ha reducido a la definición de categorías de carácter meramente cuantitativo, las cuales, además, no repercuten en un régimen diferenciado.

El marco normativo no ha tomado en cuenta, ni la totalidad de los factores de categorización, ni ninguno de los factores de diferenciación que establece la Carta Constitucional para el funcionamiento de las diferentes categorías. La norma principal, que es la Ley 136 de 1994, introdujo las bases de categorización territorial, las cuales, aunque han sido objeto de ajustes mediante otras leyes, no han cambiado ni el número y la denominación de las categorías, ni los criterios de categorización. Estas normas, que son la Ley 617 de 2000 y la Ley 1551 de 2012, utilizan, en el caso de la primera, solamente a la población y los recursos como criterios de categorización, igual que lo hizo la Ley 136; y en el caso de la segunda, aunque plantea que 
los criterios de categorización son los cuatro que establece la Constitución, termina utilizando los mismos que la 617. De igual manera, ninguna de estas normas desarrolla regímenes diferenciados para el gobierno, la organización y las funciones, ya que aunque la Ley 1551 abre la posibilidad para que, en el plazo de dos años, se expida una ley que reglamente esta materia, sus desarrollos no se han concretado hasta el momento.

La Ley 136, igual que lo repite la 617, estableció siete categorías que permiten ubicar a los municipios en la categoría especial y en las categoría primera a sexta. Los criterios de categorización, sobre la base del número de habitantes y los ingresos corrientes de libre destinación, permiten clasificar a los municipios de mayor a menor.

\begin{tabular}{|l|l|l|}
\hline \multicolumn{1}{|c|}{ Categoría municipal } & \multicolumn{1}{|c|}{$\begin{array}{c}\text { Población } \\
\left(\text { n. }^{\text {o de habitantes })}\right.\end{array}$} & \multicolumn{1}{|c|}{$\begin{array}{c}\text { Ingresos corrientes de libre } \\
\text { destinación (SMLM) }\end{array}$} \\
\hline Especial & $>=500.001 \mathrm{Hab}$ & $>400.000$ \\
\hline Primera & $100.001-500.000$ & $100.000-400.000$ \\
\hline Segunda & $50.001-100.000$ & $50.000-100.000$ \\
\hline Tercera & $30.001-50.000$ & $30.000-50.000$ \\
\hline Cuarta & $20.001-30.000$ & $25.000-30.000$ \\
\hline Quinta & $10.001-20.000$ & $15.000-25.000$ \\
\hline Sexta & $=<10.000$ & $=<15.000$ \\
\hline
\end{tabular}

Fuente: Ley 617 de 2000.

Sin embargo, la misma norma establece que un municipio o distrito que deba clasificarse en una categoría de acuerdo con su población, pero cuyos ingresos no correspondan con esta categoría, deberá clasificarse en la categoría correspondiente a la de sus ingresos corrientes. Es decir que el criterio principal de categorización son los recursos, en tanto el mismo no está supeditado a la población. De igual manera, la norma obliga a descender de categoría a las entidades que superen los porcentajes máximos de gastos de funcionamiento permitidos.

\begin{tabular}{|l|l|}
\hline \multicolumn{1}{|c|}{ Categoría } & \multicolumn{1}{c|}{ Límite } \\
\hline Especial & $50 \%$ \\
\hline Primera & $65 \%$ \\
\hline Segunda y tercera & $70 \%$ \\
\hline Cuarta, quinta y sexta & $80 \%$ \\
\hline
\end{tabular}

Fuente: Ley 617 de 2000.

Por su parte, la Ley 1551 de 2012, que se supone modifica lo que establece la Ley 617, menciona como criterios de clasificación de los municipios los cuatro que menciona la Constitución, pero en la descripción de cada una de 
las categorías incluye solo tres, la población, los recursos y la importancia económica, dejando de lado, aunque acabe de mencionarla, la situación geográfica. Adicionalmente, el criterio de importancia económica no es claro en tanto coincide con las siete categorías que ya existían desde la Ley 136 de 1994.

\begin{tabular}{|c|c|c|c|c|}
\hline Grupo & $\begin{array}{l}\text { Categoría } \\
\text { municipal }\end{array}$ & $\begin{array}{c}\text { Población } \\
\text { (n. }{ }^{\circ} \text { de habitantes) }\end{array}$ & $\begin{array}{l}\text { Ingresos corrientes } \\
\text { de libre destinación }\end{array}$ & $\begin{array}{l}\text { Importancia } \\
\text { económica }\end{array}$ \\
\hline \multirow{2}{*}{$\begin{array}{l}\text { Primer grupo: } \\
\text { municipios grandes }\end{array}$} & Especial & $>=500.001$ & $>400.000$ & Grado uno \\
\hline & Primera & $100.001-500.000$ & $100.000-400.000$ & Grado dos \\
\hline \multirow{3}{*}{$\begin{array}{l}\text { Segundo grupo: } \\
\text { municipios } \\
\text { intermedios }\end{array}$} & Segunda & $50.001-100.000$ & $50.000-100.000$ & Grado tres \\
\hline & Tercera & $30.001-50.000$ & $30.000-50.000$ & Grado cuatro \\
\hline & Cuarta & $20.001-30.000$ & $25.000-30.000$ & Grado cinco \\
\hline \multirow{2}{*}{$\begin{array}{l}\text { Tercer grupo: } \\
\text { municipios básicos }\end{array}$} & Quinta & $10.001-20.000$ & $15.000-25.000$ & Grado seis \\
\hline & Sexta & $=<10.000$ & $=<15.000$ & Grado siete \\
\hline
\end{tabular}

Fuente: elaborado con base en la Ley 1551 de 2012.

Además, esta ley incluye la misma directriz de la Ley 617 en la que se ordena que si un municipio o distrito, de acuerdo con su población, debe clasificarse en una categoría pero sus ingresos no corresponden con esta, deberá clasificarse en la categoría que corresponda a sus ingresos corrientes. Por ende, esta norma termina estableciendo el nivel de recursos como único criterio de categorización.

La consideración del municipio como una entidad que debe ser evaluada por su capacidad económica, sin considerar sus demás atributos, se ilustra también en estas dos normas al endurecer de manera progresiva los requisitos para la creación de nuevas entidades del nivel local. Debido a las dificultades fiscales que enfrentan hoy día los municipios, las normas han buscado limitar al máximo la creación de este tipo de entidades. Ante una consideración de los municipios como entidades "ricas" o "pobres", la mejor opción es limitar la aparición de más municipios "pobres".

Un aspecto importante por resaltar de la Ley 1551 y que la diferencia de las normas anteriores es que introduce de manera tímida una especie de supracategorización, mediante la cual reúne a las entidades de nivel local en grupos de municipios grandes, intermedios y básicos. El propósito de esta clasificación es el establecimiento de regímenes diferenciados en materia de organización, gobierno y administración. Con esto, la ley estaría estableciendo en la práctica solo tres categorías de municipios, ya que, como lo establece la Constitución, los regímenes diferenciados deben estar ligados a cada categoría municipal y no a grupos de categorías. 


\subsection{Marco normativo de la categorización departamental}

La categorización departamental ha recibido el mismo tratamiento que en el caso de los municipios, ya que con la Ley 617 de 2000 se establecieron cuatro categorías tomando en cuenta la población y los recursos, con el fin de clasificarlos de mayor a menor. Las situaciones excepcionales descritas para los municipios se hicieron extensivas a los departamentos, ya que los recursos propios constituyen el criterio dominante para la categorización, y opera una regla similar en términos de límites para gastos de funcionamiento en cada categoría.

\begin{tabular}{|l|l|l|}
\hline $\begin{array}{c}\text { Categoría } \\
\text { departamental }\end{array}$ & \multicolumn{1}{|c|}{$\begin{array}{c}\text { Población } \\
\text { (n. }{ }^{\circ} \text { de habitantes) }\end{array}$} & $\begin{array}{c}\text { Ingresos corrientes de libre } \\
\text { destinación (sMLM) }\end{array}$ \\
\hline Especial & $>2.000 .00$ & $>600.000$ \\
\hline Primera & $700.001-2.000 .000$ & $170.000-600.000$ \\
\hline Segunda & $390.001-700.000$ & $122.001-170.000$ \\
\hline Tercera & $100.001-390.000$ & $60.001-122.000$ \\
\hline Cuarta & $=<100.00$ & $=<60.000$ \\
\hline
\end{tabular}

Fuente: elaborado con base en la Ley 617 de 2000.

Por su parte, las nuevas consideraciones sobre importancia económica introducidas en la categorización municipal no son aplicables al departamento, en tanto su desarrollo se introdujo en la Ley 1551 de 2012, expedida para "modernizar la organización y el funcionamiento de los municipios". Igual sucede con la disposición sobre creación de nuevas entidades territoriales, ya que esta posibilidad fue introducida, para los departamentos, apenas en el año 2011, con la Ley 1454, y todavía no existe una reglamentación precisa sobre cantidad de recursos y/o población.

\section{ALTERNATIVAS DE LA CATEGORIZACIÓN MUNICIPAL}

\subsection{Criterios de categorización municipal}

Aunque los contenidos tanto de los factores de categorización como de los criterios para la definición de regímenes diferenciados no se encuentran descritos en la Constitución, ya que esto le corresponde a la ley, es fácil suponer cuáles podrían ser las distintas alternativas que cabría considerar en cada uno de ellos. Por ejemplo, en las normas existentes la población es considerada como una cifra. Sin embargo, dicha cifra puede desagregarse atendiendo a los intereses de la gestión pública, en términos de género, grupos de edades, nivel socioeconómico, cultura, etnia, así como de los niveles de cobertura de bienes y servicios públicos, NBI, entre otros. 
El nivel de desagregación de este criterio a la hora de pensar en la categorización municipal dependerá de los propósitos de esta; lo cierto es que el mismo debe articular la dimensión cualitativa. De hecho, una descripción más clara de la población aporta mayor información cuando se articula con el nivel de los recursos fiscales de la respectiva entidad. En este sentido sería claro cuál es la capacidad real que tiene una entidad para asumir responsabilidades públicas, dadas las particularidades de cobertura y calidad de los servicios para su población. Esto permitiría evaluar también el nivel requerido de participación de otros niveles, como el departamental y el nacional. El factor de recursos también podría tomar en consideración el nivel de endeudamiento de un municipio y la participación de las fuentes externas dentro de su presupuesto anual. De esta forma sería posible establecer categorías a partir de un agregado mínimo de funciones para cualquier administración local.

Por su parte, la importancia económica, considerada actualmente como la cantidad de PIB que aporta determinada entidad frente a la producción bruta nacional, podría adquirir un carácter cualitativo. En tal sentido se podría clasificar a los municipios por su vocación económica, en materia industrial, a nivel micro, mediano y macro; minero y energético; de servicios, financieros, turísticos, comercial, educativo; agropecuario, forestal, pesquero, entre otros.

Finalmente, la situación geográfica, al permitir ubicar a una entidad en una zona costera, fronteriza, selvática, de llanura, montañosa, etc., puede aportar información en diferentes aspectos de interés para el Estado. Este factor se articula con la vocación económica, determinada por los factores geográficos y climáticos, y en materia social puede ser trascendental para evaluar la capacidad fiscal del Estado en términos del acceso a los territorios, atención social determinada por factores culturales y étnicos, densidad poblacional, entre otros. Además, la situación geográfica es el principal factor para determinar la importancia ambiental de un territorio y su pertenencia a regiones ambientalmente relevantes.

\subsection{Factores de diferenciación del régimen municipal}

Una vez definidas las categorías territoriales a partir de la combinación de los factores descritos, la Constitución de 1991 establece que las distintas categorías resultantes deberían diferenciarse en términos de administración, organización y gobierno.

El componente más importante de los tres es el que se refiere a la $a d m i$ nistración; de hecho, aunque nunca se concretó en la práctica, este se incluyó como el único factor diferenciador del funcionamiento de los municipios durante la vigencia de la Constitución anterior a 1991. Su importancia radica en que la ubicación de cada municipio en determinada categoría debería influir en la cantidad y clase de funciones administrativas, referidas principalmente a las responsabilidades sectoriales y territoriales. Las distintas categorías 
deberían asumir las funciones partiendo de un nivel mínimo hasta llegar al agregado total de funciones que corresponde a los municipios en la actualidad.

Las funciones de las entidades territoriales se pueden agrupar actualmente en tres conjuntos visiblemente diferenciados, pero que se han asignado a todas por igual. En primer lugar, están aquellas relacionadas con los diferentes sectores de inversión pública, las cuales se han desarrollado mediante una o más leyes ordinarias para cada sector, en las que se define cuál es la participación de cada uno de los niveles del Estado. Estas funciones representan el mayor desafío para los recursos propios, en especial de los entes locales. En segundo lugar, están los desarrollos normativos tendientes al mejoramiento de la gestión de las organizaciones públicas dentro de la lógica de la Nueva Gestión Pública. Estas normas presionan la capacidad de las entidades, en especial lo relacionado con las capacidades técnicas y administrativas de que disponen las plantas de personal, las cuales se financian principalmente con recursos propios. El buen desempeño de estas funciones es trascendental, debido a que constituyen la base sobre la que se soporta la programación y desarrollo del primer grupo de responsabilidades.

Finalmente, están las funciones relacionadas con la asignación y distribución de las transferencias provenientes del nivel nacional, que inicialmente se reglamentaron mediante la Ley 60 de 1993, la cual fue derogada por la Ley 715 de 2001, y esta a su vez fue complementada mediante la Ley 1176 de 2007. Estas normas recogen las funciones sectoriales de las entidades territoriales, que ya se mencionaron en el primer grupo de normas; sin embargo, por tratarse de normas referidas al Sistema General de Participaciones, su énfasis se encuentra puesto en los sectores, que de acuerdo con las directrices nacionales deben ser los destinatarios de estos recursos a nivel territorial. En este sentido, aunque estas normas destinan unos pocos recursos para libre inversión, y se pueden orientar tomando en cuenta criterios de priorización para los diferentes sectores en los que tienen competencias las entidades territoriales, las normas en cuestión establecen que la asignación de los recursos debe dirigirse principalmente a educación, salud y agua potable. El ejercicio de estas funciones con recursos provenientes de transferencias les otorga a las entidades territoriales un carácter de desconcentración y no de descentralización territorial, y le da mayor protagonismo al departamento frente al municipio (Duque, 2010).

En la mayoría de los casos los recursos propios del nivel local se destinan principalmente para cubrir los gastos de funcionamiento de la administración, es decir, para el cumplimiento del segundo grupo de funciones asignadas. Aunque la Ley 617 de 2000 estableció unos límites máximos para estos gastos con el fin de procurar un mínimo para inversión social, de todas maneras los recursos propios no permiten un amplio nivel de maniobra. Si a los recursos propios les restamos los gastos de funcionamiento, los recursos disponibles para inversión se reducen a un 20\%, para los municipios de categorías cuarta, 
quinta y sexta, los cuales suman cerca del $90 \%$ de los municipios del país (Contaduría General de la Nación, 2016). Es decir que las funciones que mayor peso generan para las entidades territoriales, y de las que depende la calidad de vida de las poblaciones, no se pueden garantizar, con algunas excepciones, con recursos propios, y solo algunas de las funciones relativas a inversión social se pueden garantizar mediante el sistema de transferencias.

\section{RELACIÓN ENTRE FUNCIONES Y RECURSOS}

DE LOS MUNICIPIOS EN COLOMBIA

\begin{tabular}{|c|c|c|c|}
\hline & $\begin{array}{l}\text { Funciones sectoriales } \\
\text { del municipio (inversión) }\end{array}$ & $\begin{array}{c}\text { Funciones de gestión } \\
\text { organizacional } \\
\text { (funcionamiento) }\end{array}$ & $\begin{array}{c}\text { Funciones sectoriales } \\
\text { financiadas con transferencias } \\
\text { (funcionamiento) }\end{array}$ \\
\hline 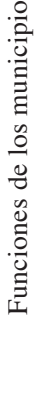 & $\begin{array}{l}\text { - Salud } \\
\text { - Educación } \\
\text { - Agua potable y saneamiento } \\
\text { básico } \\
\text { - Ambiente } \\
\text { - Cultura, recreación y deporte } \\
\text { - Infraestructura y transporte } \\
\text { - Turismo } \\
\text { - Desarrollo económico } \\
\text { - Desarrollo agropecuario } \\
\text { - Juventud } \\
\text {-Otros }\end{array}$ & $\begin{array}{l}\text { - Planeación } \\
\text { - Presupuesto } \\
\text { - Gestión del personal } \\
\text { - Control interno } \\
\text { - Contratación pública } \\
\text { - Gestión de proyectos de } \\
\text { inversión } \\
\text { - Desarrollo organizacional } \\
\text { - Gestión de la calidad } \\
\text { - Ordenamiento Territorial }\end{array}$ & $\begin{array}{l}\text { - Salud } \\
\text { - Educación } \\
\text { - Agua potable y saneamiento } \\
\text { básico }\end{array}$ \\
\hline 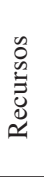 & $\begin{array}{l}\text { Recursos propios: } \\
\text { - Categoría especial: } 50 \% \\
\text { - Categoría } 1 .^{\text {a: }: 35 \%} \\
\text { - Categoría } 2 .^{\text {a: }: 30 \%} \\
\text { - Categorías } 4 .^{\text {a }}, 5 .^{\text {a }} \text { y } 6 .^{\text {a }:}: 20 \%\end{array}$ & 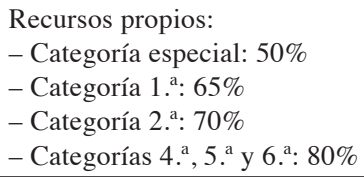 & $\begin{array}{l}\text { Transferencias del sistema } \\
\text { general de participaciones. }\end{array}$ \\
\hline
\end{tabular}

Fuente: elaboración propia, tomando en cuenta las leyes 617 de 2000, 715 de 2001 y 1176 de 2007.

Aunque durante la década anterior se presentó un aumento importante tanto de los ingresos de las entidades territoriales como del gasto, en especial para el caso de los municipios, donde se observan, de manera agregada, incrementos de ingresos propios superiores al $80 \%$, las fuentes exógenas siguen siendo la principal fuente de recursos, en especial las provenientes del SGP (Zapata, 2010). De acuerdo con los informes sobre desempeño fiscal de los municipios y departamentos del DNP, entre 2000 y 2013 se evidenció que los ingresos propios de los municipios en relación con el total de los recursos recibidos por las entidades territoriales son, en términos proporcionales, mucho más bajos en los municipios de menor tamaño poblacional y menores ingresos, mientras que son más importantes en los de categoría especial, primera y segunda. 
PARTICIPACIÓN DE LAS FUENTES ENDÓGENAS Y EXÓGENAS

EN LOS INGRESOS MUNICIPALES 2009-2013

\begin{tabular}{|l|c|c|c|}
\hline Categoría & $\begin{array}{c}\text { Porcentaje de } \\
\text { municipios y distritos }\end{array}$ & $\begin{array}{c}\text { Fuentes endógenas } \\
\text { promedio \%): ingresos } \\
\text { tributarios y no } \\
\text { tributarios }\end{array}$ & $\begin{array}{c}\text { Fuentes exógenas } \\
\text { (promedio \%): } \\
\text { transferencias, sGP, } \\
\text { regalías, cofinanciación, } \\
\text { otros }\end{array}$ \\
\hline Especial & 0,6 & 56,6 & 43,2 \\
\hline Primera & 2,1 & 38,4 & 61 \\
\hline Segunda & 1,8 & 32 & 68 \\
\hline Tercera & 1,3 & 34,8 & 65,2 \\
\hline Cuarta & 3 & 17,2 & 83,2 \\
\hline Quinta & 3,4 & 28 & 71,4 \\
\hline Sexta & 87,8 & 14,2 & 85,4 \\
\hline
\end{tabular}

Fuente: DNP, 2009, 2010, 2011, 2012, 2013.

En un proceso de categorización acorde a las condiciones de los municipios, sería necesario partir de la cantidad de recursos disponibles para inversión. También se deberían considerar las combinaciones funcionales más pertinentes hasta llegar a niveles cada vez más complejos y diversos. En este sentido, el lenguaje actual relativo a la existencia de municipios "pequeños", "débiles" y "pobres" debería remplazarse por otro tipo de calificativos más acordes con su nivel de importancia o caracterización.

Una vez establecidas las características y la cantidad de las funciones correspondientes a cada una de las categorías municipales, se debe pensar en la forma de organización interna de las entidades que se ubiquen en cada categoría. Esto con el fin de que esta organización esté acorde con los propósitos planteados, teniendo claro un nivel de flexibilidad aceptable para que cada entidad territorial pueda reflejar en este criterio sus particularidades.

Finalmente, la categorización territorial podría derivar en la diferenciación de las entidades en términos de gobierno, es decir, el tipo de autoridades que debería corresponder a cada categoría. Esto implicaría romper con la regla actual que asigna el mismo tipo de autoridades y la misma forma de designación de estas a nivel territorial. Se podría pensar que dichas autoridades están consideradas para ámbitos de actuación en los que la entidad tiene que asumir la totalidad de funciones del nivel territorial respectivo. Por lo tanto, otras categorías con menos funciones o con funciones de diferente nivel de complejidad podrían contar con otras alternativas en materia de autoridades, de selección de las mismas o de mecanismos para la toma de decisiones.

Dado lo anterior, se podría considerar la eliminación de alguna de estas autoridades o de las dos (alcalde y concejo municipal), considerar la participación ciudadana en remplazo de alguna de ellas o de ambas y/o exigir un 
perfil más técnico o más político para estas autoridades donde lo requiera la categoría. Esto podría conducir a considerar la combinación o el uso exclusivo del concurso de méritos o la elección popular en la selección. Incluso, se podrían considerar mecanismos de valoración ética y moral, de tal manera que sea posible descartar alternativas inconvenientes o peligrosas para el interés público.

\section{ALTERNATIVAS DE LA CATEGORIZACIÓN DEPARTAMENTAL}

Una vez resuelto el diseño municipal es necesario avanzar hacia arriba en el edificio institucional con el propósito de replantear el papel del departamento e incluso de la nación en la lógica intergubernamental. En una nueva lógica de categorización, los departamentos podrían llegar a asumir funciones que hoy son de naturaleza local, con el fin de complementar la actuación de aquellas categorías municipales que así lo requieran. Esto permitiría resolver en primer lugar las imprecisiones del diseño normativo en donde se le asignan al departamento las mismas funciones que a los municipios, y por otro, precisar su papel como unidad desconcentrada del nivel nacional en la ejecución de los recursos de transferencias. Asimismo, se deberían replantear las funciones departamentales atendiendo a la lógica del nivel intermedio, a partir, incluso, de los mismos o distintos factores considerados desde la Constitución para la categorización municipal, dando lugar de esta manera a una nueva categorización departamental.

A diferencia de lo que sucede con los municipios, ni la Constitución de 1991, ni ninguna otra norma anterior o actual hace referencia al deber ser de la categorización de los departamentos. Como es de esperarse, y por tratarse de entidades territoriales que se componen, territorialmente, de otras entidades, los municipios y distritos, las condiciones o factores a tomar en cuenta para su categorización deben diferenciarse en mayor o menor medida de aquellos factores establecidos para el nivel local. Para el caso de los departamentos podrían resultar pertinentes los factores relacionados con la cantidad de recursos, la importancia económica, y la situación geográfica.

El factor poblacional cuya lógica se debe ubicar preferentemente en el municipio, por ser la entidad responsable del vínculo directo con la sociedad, podría ser un factor de categorización departamental en los casos de debilidad local evidente. Sin embargo, se debería establecer como criterio de categorización departamental uno que permita caracterizar sus municipios, por ejemplo, por la cantidad de municipios que existe en su interior, las categorías de estos municipios, así como la existencia de territorios que no pertenecen a ninguna entidad territorial del nivel local, entre otros. A partir de lo anterior sería posible clasificar a los departamentos por sus características predominantes en términos económicos, ambientales, políticos, fiscales, entre otros, y finalmente, por las características predominantes en materia municipal. 
La diferenciación del régimen departamental se podría reflejar en aspectos similares a los de los municipios, por ejemplo, en materia de funciones. Como veíamos en relación con los municipios, aquellas entidades que solo alcancen a cumplir las funciones básicas deberán ser complementadas con la participación de los departamentos. Esto conduciría a la asignación de funciones de diferente nivel de complejidad a nivel intermedio, dependiendo de las necesidades de sus entidades locales. Incluso habría casos en los que el departamento tendría que asumir las funciones municipales desde el nivel básico, por ejemplo cuando se trate de administrar territorios que no pertenecen a ningún municipio o distrito, como sucede actualmente por la existencia de los denominados corregimientos departamentales, presentes en los departamentos de Amazonas, Guainía, Vaupés y Nariño (Ley 1551 de 2012). Esta particularidad se podría resolver si eventualmente fueran creadas las entidades territoriales indígenas, ya que estos territorios se componen en su mayoría por resguardos indígenas.

Además de las funciones de prestación directa, el papel del departamento debería configurarse en atención a las funciones que directamente lo vinculen con sus municipios en tanto realidades institucionales. En este sentido, deberían definirse sus obligaciones pensando en lo que cada una de las categorías de sus municipios requiere en materia de asistencia y apoyo técnico y administrativo, así como de coordinación intermunicipal. Su nivel de funciones, articulado con su capacidad fiscal, debería dar lugar a una configuración organizacional interna particular pensando en cuál sería la mejor forma de atender sus nuevas obligaciones. En lo que se refiere al gobierno, es posible incluso volver a abrir el debate sobre la validez de mantener la elección popular tanto de gobernadores como de las asambleas departamentales, dadas las rupturas que genera la influencia de la dinámica partidista y política en el manejo de los asuntos y los recursos públicos en estos territorios en la actualidad. En especial tomando en cuenta que estas entidades deberían combinar tanto factores técnicos como de articulación intergubernamental con la Nación y los municipios (Duque, 2010).

También, es factible implementar una fórmula intermedia entre concurso de méritos y elección popular igual que en caso del nivel local. Incluso, cabría la consideración de replantear la eliminación de alguna de estas dos autoridades o la eliminación de las dos ya que habría departamentos cuyo papel, en relación con las funciones de prestación directa de servicios, se reduzca considerablemente y se concentre en la realización de funciones de apoyo y asistencia técnica municipal y coordinación con el nivel nacional. En los casos de ausencia de la asamblea departamental es posible pensar en procesos de toma de decisiones que hagan posible la participación directa de los municipios, y que permitan que estos, a través de sus alcaldes o representantes, participen en la definición de los programas y proyectos más importantes. Lo anterior se asemejaría a una 
figura de asociatividad, pero lograda en articulación con otros municipios y con su departamento.

\section{DISEÑO MUNICIPAL Y CATEGORIZACIÓN A NIVEL INTERNACIONAL}

Igual que en Colombia, a nivel mundial la tendencia muestra que las reformas constitucionales o legales han transferido la responsabilidad de la mayoría de los servicios básicos a los gobiernos locales. Esta tendencia ha estado fundada en la necesidad de reconocer la importancia del nivel local para la calidad de vida de las poblaciones, y también del derecho de estas para participar en los procesos de decisión pública. No obstante lo anterior, se ha hecho evidente que la descentralización ha generado presiones importantes en la capacidad de respuesta del nivel municipal (CGLU, 2013).

En la mayoría de los países de América Latina los municipios pueden prestar cualquier servicio que no esté asignado a otro nivel de gobierno, sin embargo, no es posible generalizar sobre el diseño de las funciones municipales en la región. Algunas de las funciones son obligatorias, como es el caso de los servicios de recolección de basuras, mantenimiento de carreteras, parques, plazas de mercado y mataderos, igual que funciones administrativas como registro civil, planeación urbana, usos del suelo y permisos de vivienda. Las funciones que se comparten con los otros niveles en algunos casos son voluntarias, estas funciones se refieren a servicios sociales como la educación básica, y los servicios de atención primaria de salud, agua y alcantarillado (CGLU, 2011). En Colombia, aunque las funciones de los municipios en general son obligatorias, en la práctica muchas de ellas permanecen pendientes por la falta de recursos; en particular se trata de aquellas funciones que demandan inversión para infraestructura. Los servicios de salud, educación, agua y alcantarillado están asignados a los tres niveles de gobierno, sin embargo, en la práctica los municipios pequeños terminan sujetos a los recursos y por ende las directrices que vienen desde la nación y el departamento.

En Europa la asignación de las responsabilidades de gasto a las autoridades locales involucra dos dimensiones. Primero, cuentan con una "cláusula general de competencias" consagrada en la Carta Europea de la Autonomía Local, que les permite intervenir en cualquier asunto de interés público local, siempre y cuando no afecte el ámbito de competencia de otro nivel de gobierno y no contradiga el marco legal. Este principio está consagrado en el artículo 4 de la Carta Europea de la Autonomía Local. En segundo lugar ejercen las competencias que les asignan las leyes y que generalmente representan la mayor parte del gasto de los gobiernos locales. De igual manera pueden asumir funciones delegadas desde el nivel nacional (Velasco, 2010). Las funciones asignadas al nivel local incluyen generalmente la educación primaria, en términos de construcción, operación y mantenimiento de las escuelas, la infraestructura y la planificación urbana, la recolección y el 
tratamiento de residuos, la distribución de agua potable y el tratamiento de aguas residuales, y la prestación de los servicios sociales, como la protección de la infancia, la ayuda a los ancianos y a los discapacitados (CGLU, 2011).

Sumado a la diversidad de posibilidades funcionales, en la mayoría de los casos la descentralización se ha realizado dentro de una lógica de uniformidad normativa. Sin embargo, aunque el municipio es, en general, el depositario de los servicios básicos, en la práctica se han establecido alternativas que sirven de atenuante a las debilidades latentes de este nivel territorial. En términos generales los municipios comparten las responsabilidades de la gestión con los gobiernos intermedios y nacionales (CGLU, 2013; Massiris y otros, 2012).

Aunque el nivel local tiene funciones asignadas, son los otros niveles, en especial el nivel nacional, los que siguen garantizando su provisión (CGLU, 2013). Esto refleja la tendencia del nivel nacional a mantener el control sobre los territorios, tanto en la toma de decisiones como en la ejecución de los recursos, gracias a la existencia de un diseño normativo, en algunos casos restringido y en otros bastante difuso. Aunque se ha avanzado en la descentralización de funciones, no ha sucedido lo mismo en la asignación de suficientes recursos propios para respaldarlas, condenando a los municipios a depender de las transferencias nacionales (Eguino, 2010; Quenan, 2014).

\subsection{Principio de subsidiariedad}

Además de un diseño institucional donde las diferentes funciones se han distribuido, la mayoría de las veces, de forma concurrente y complementaria, los diferentes países han incluido como posibilidad de articulación intergubernamental el principio de subsidiariedad para aquellas funciones que son propias de la entidad territorial. Este principio se orienta a la consideración de que la autoridad organizadora deba ser asumida por el nivel de gobierno más eficiente y que esté lo más cerca posible de las personas (CGLu, 2013). En Colombia este principio tiene la particularidad de que aunque se reconozca la existencia de funciones que son exclusivas de un nivel de gobierno determinado, si los defectos en la capacidad administrativa de una entidad específica son tan evidentes, es necesario que la misma solicite el apoyo de cualquiera de las entidades del nivel superior. Dicho apoyo, el cual no implicaría que el nivel superior adquiera la función sino que la asuma mientras la entidad débil logra vencer sus debilidades, solo se justifica cuando esta última lo solicita, evitando de esta forma cualquier intromisión no deseada que pueda ir en contravía de la autonomía territorial (Sentencia C-004-93).

\subsection{Esquemas de asociatividad territorial}

Otra alternativa para que los municipios soslayen sus debilidades internas en términos administrativos y fiscales y puedan cumplir las responsabilidades 
asignadas son los esquemas asociativos. En el caso de Europa las reformas municipales de finales del siglo xx derivaron en la mayoría de los casos en la fusión de municipios. Sin embargo, países como Alemania, Bélgica, Grecia, Dinamarca y Reino Unido, que redujeron el número de municipios en aras de mejorar su capacidad de respuesta, también incluyeron diferentes alternativas que permiten que los municipios trabajen conjuntamente. En algunos casos (Francia, Italia, Hungría y España) estas formas de cooperación intermunicipal se han concebido, incluso, como alternativas a la fusión de municipios (CGLU, 2008).

Un caso particular de asociatividad son los Kreise alemanes, que son considerados entidades locales de ámbito supramunicipal y que tienen el doble carácter de corporación territorial y el de asociación de municipios. Como división territorial ejercen las competencias del Estado que se les han asignado y delegado. Como asociación ejercen aquellas tareas y funciones que exceden la capacidad de gestión de los municipios más débiles. Los municipios que no están integrados a los Kreise son ciudades y asumen directamente las funciones que normalmente se les asignan a los primeros (Velasco y otros, 2010). Además de los Kresie el ordenamiento territorial alemán admite otro tipo de figuras de asociatividad entre municipios, y también de trabajo conjunto mediante figuras, como el convenio, que no requieren la conformación de una entidad; igual que sucede en Italia y el Reino Unido.

En el caso de América Latina, diferentes países también han promovido la conformación de mancomunidades y otras formas de asociatividad con el fin de resolver el problema de la limitación de la capacidad de respuesta de los pequeños municipios. En tal sentido países como Bolivia y Argentina superan cada uno las 70 mancomunidades, orientadas a la promoción del desarrollo regional, turístico y ambiental. En Ecuador se cuentan 20 mancomunidades, en Chile 60 asociaciones subregionales y sectoriales de municipios, y en Colombia 44 asociaciones de municipios orientadas a la prestación de servicios públicos, ejecución de obras o cumplimiento de funciones administrativas (CGLU, 2008).

En nuestro país, además de las asociaciones de municipios que se encuentran consideradas desde 1968, así como las áreas metropolitanas, la Ley 1454 de 2011 amplió la cantidad de entidades asociativas territoriales en las que pueden participar los municipios y los distritos y que se pueden conformar con propósitos y alcance temporal distintos. Esta ley amplió las posibilidades de asociatividad también para el nivel intermedio, ya que además de las regiones administrativas y de planificación creadas con la Constitución Política de 1991, se incluyeron otras alternativas como la región administrativa y de planificación especial (RAPE), las asociaciones de departamentos y las regiones de planeación y gestión. Estas últimas también pueden incluir al nivel local (Duque, 2012). 


\subsection{Categorización municipal}

La categorización municipal solo se encuentra considerada en las constituciones de dos de los países estudiados, ambos de carácter federal. El primer caso es Venezuela, cuya Constitución en el artículo 169 establece la necesidad de generar diferentes regímenes para el nivel local en términos de organización, gobierno y administración, incluyendo el tema de competencias y recursos. Estos diferentes regímenes deben tomar en cuenta las condiciones de población, desarrollo económico, capacidad para generar ingresos propios, situación geográfica, elementos históricos y culturales y otros factores relevantes.

Aunque estos factores tanto de categorización como de diferenciación del régimen municipal son prácticamente los mismos que en Colombia, y se encuentran también referidos en la Ley Orgánica de Poder Público Municipal (arts. 6 y 47), la misma Constitución establece restricciones para su materialización. En primer lugar, la Carta establece las mismas autoridades para todos los municipios, y en segundo término enumera las competencias y los ingresos municipales, dejando muy poco margen tanto para la Asamblea Nacional como para los consejos legislativos estadales en la definición de regímenes distintos (González, 2000). De hecho, reformas a normas tan importantes como la Ley Orgánica de Descentralización, Delimitación y Competencias del Sector Público (LOD), en lugar de avanzar en la diferenciación de regímenes territoriales, han derivado en la recentralización de competencias que habían sido cedidas anteriormente a los estados.

El segundo caso identificado es Argentina, cuyas constituciones provinciales, "además de considerar distintas categorías de municipios, incluyen otras formas de gobierno local sin jerarquía municipal" (Iturburu, 2009, p. 58 ), las cuales pueden ser dependientes "de la provincia (por lo tanto son 3 . nivel de gobierno) o depender del municipio (ya sea como meras delegaciones administrativas o con posibilidad de elegir autoridades, lo que constituiría un 4. ${ }^{\circ}$ nivel de gobierno)" (Iturburu, 2009, p. 64). En relación con los municipios, las provincias pueden definir una (4 provincias), dos (5 provincias), tres ( 7 provincias) o cuatro (5 provincias) categorías municipales. De las 24 provincias, 18 establecen como criterio de categorización la cantidad de población; las otras provincias utilizan como criterios la cantidad de electores y otros criterios como ser capital, división territorial de la provincia o ser centro urbano (Iturburu, 2007, p. 27).

La categorización deriva en una diferenciación en materia de asignación de las funciones municipales que para cada provincia es particular, y bastante variada, impidiendo establecer generalizaciones. Sin embargo, no se evidencia la existencia de asignación incremental de funciones atendiendo al tamaño poblacional o a otro tipo de característica local en ninguna de las provincias (Iturburu, 2007). 
En los demás países, en lugar de en la categorización municipal, tal como se ha vislumbrado en nuestra Constitución, se ha avanzado en el reconocimiento de dos clases de entidades de nivel local, sujetas principalmente a las diferencias en términos de cantidad de población. Esta diferenciación se deriva de las tendencias generalizadas a nivel mundial en las que abundan los municipios pequeños en población y sobresalen algunas pocas ciudades por contar con niveles poblacionales muy grandes y en constante ascenso. Aunque en los países de Europa del Este y del Norte, descartando al Reino Unido, el diseño normativo se caracteriza por un estatuto único para todos los municipios, en otros territorios, como el Reino Unido, Europa Central y Oriental, a partir de Alemania, las ciudades cuentan con un régimen particular (CGLu, 2008). De hecho, debido a su tamaño y sus particularidades frente al municipio tradicional, en algunos casos (Reino Unido, Alemania, y en menor medida España) las ciudades no se integran al segundo nivel territorial. Incluso, algunas ciudades capitales, como Londres y Berlín, han adquirido el doble carácter, tanto de ente local de primer nivel como de segundo nivel o nivel intermedio, dependiendo del país (Velasco y otros, 2010).

Por su parte, en América Latina más de la mitad de la población habita en ciudades que superan el millón de habitantes, cuyo crecimiento ha derivado en fenómenos importantes de conurbación. Esta circunstancia ha conducido, para el caso de las ciudades capitales, al reconocimiento de estructuras metropolitanas que abarcan varios municipios, y en algunos casos atraviesan varios estados o provincias. Los casos más sobresalientes son los de la Ciudad de México y su Zona Metropolitana -definida en 1995- que abarca 41 municipios ubicados en dos estados, además del Distrito Federal; Buenos Aires, que cubre el territorio de la ciudad autónoma de Buenos Aires y el de 32 municipios de la provincia de Buenos Aires; el Gran Santiago (Chile), que integra 39 municipios, y São Paulo (Brasil), que cuenta con 39 prefeituras (CGLU, 2008). Sin embargo, "si bien varias ciudades capitales de Latinoamérica poseen regímenes especiales -como Bogotá, Buenos Aires, Caracas, Lima, México o Quito-, son pocas las que tienen un gobierno metropolitano para gestionar de modo integrado el territorio urbano", entre las que se cuentan Lima, Quito y Caracas (CGLU, 2008, p. 109).

Además de la diferenciación de regímenes orientada a las ciudades capitales, solo en un caso se encontró referencia constitucional a la diferenciación en dos clases de municipios dependiendo del tamaño poblacional. Este es el caso de Chile, que a través de la Ley Orgánica Constitucional de Municipales distingue entre municipios de más de cien mil habitantes y municipios de menos de cien mil habitantes, con el fin de diferenciar la forma de organización interna de la administración. En este sentido, las comunas de más de cien mil habitantes puedan crear más secretarías para cumplir con las funciones asignadas a este nivel de gobierno, que son iguales para las dos clases de comunas. 
En el caso de Colombia, la Constitución de 1991 distingue entre municipios y distritos, las dos entidades son de nivel local y en el segundo caso se trata de municipios que han adquirido un estatus especial que debe ser reconocido por la Constitución Política. Adicionalmente, estas entidades tienen un régimen especial que prevalece sobre las disposiciones de carácter general que integran el régimen ordinario de los municipios. En el caso de Bogotá, la entidad goza de un régimen particular (Dcto. 1421 de 1993) distinto al régimen aplicable a los demás distritos del país. No obstante, en relación con los municipios y como veíamos antes, lo que prevalece es la uniformidad normativa en términos funcionales, organizacionales y de sus autoridades.

\section{CONCLUSIONES}

La descentralización territorial ha sido uno de los fenómenos más importantes dentro de la transformación reciente del Estado. En América Latina dicho fenómeno fue una reacción contra el excesivo centralismo que caracterizó al sector público durante el siglo xx, con el fin de resolver los diferentes problemas derivados del diseño anterior, asociados a la asignación de recursos, el impacto social, la legitimidad del Estado, la gestión pública, entre otros. No obstante los propósitos, los resultados del proceso están sujetos al diseño del mismo, del cual también dependen otros fenómenos de funcionamiento del Estado como son la autonomía territorial y las relaciones intergubernamentales. Los cuales se pueden ver restringidos o fortalecidos dependiendo de la forma como se conciba y se ponga en práctica la descentralización.

En Colombia, la Constitución de 1991 consideró la definición de categorías territoriales que permitieran distinguir a los municipios en términos de funciones, organización y gobierno, a partir de sus diferencias en materia de recursos, población, situación geográfica e importancia económica. Sin embargo, los desarrollos normativos solamente tomaron en cuenta los recursos como factor diferenciador y la categorización no repercutió en una distinción en las funciones, el gobierno y la organización de las entidades territoriales. En estas condiciones, la descentralización se ha construido sobre una base normativa uniforme, definida a partir del parámetro de las entidades territoriales más grandes, conviriendo a las demás en débiles al no poder igualar sus capacidades y potencialidades con aquellas. Esta situación ha impedido que particularmente los municipios, y en especial los más pequeños, hayan podido aprovechar sus ventajas y cualidades para el mejoramiento de las condiciones de sus territorios y la calidad de vida de sus poblaciones.

En diferentes regiones del mundo al nivel municipal se le ha asignado la responsabilidad en la provisión de la mayoría de los servicios sociales en este nivel del Estado. Esta tendencia, que se presenta no solo en Colombia, sino que se pudo verificar en el resto de países de América Latina y en Europa, ha coincidido con la debilidad institucional, en especial de las entidades 
más pequeñas. Para hacer frente a esta realidad la mayoría de los países han incorporado en sus diseños normativos alternativas orientadas a garantizar la adecuada provisión de los servicios sociales. Entre estas opciones se incluye la participación concurrente y complementaria de los diferentes niveles territoriales en dicha provisión; la subsidiariedad cuando una entidad territorial no pueda asumir las funciones que se le han asignado; los esquemas asociativos, que permiten la articulación entre entidades del mismo o incluso diferente nivel territorial para garantizar el cumplimiento de funciones, la construcción de obras y la prestación de servicios.

Asimismo, como tendencia generalizada, los distintos países han tenido que diferenciar entre los municipios en general y las ciudades capitales, lo cual ha dado lugar a la definición de regímenes diferenciados. En casos específicos, el régimen especial se ha extendido también a las ciudades más grandes, en particular en Europa, y solo en Perú, para América Latina, se encontró referencia constitucional sobre la diferenciación entre entidades grandes y pequeñas con fines de diferenciar la organización interna de la administración. En Colombia se avanzó recientemente en la definición de un régimen especial para los distritos.

Sin embargo, solo Argentina presenta desarrollos en materia de categorización municipal, los cuales son distintos para cada una de sus provincias debido a su naturaleza de Estado Federal. Dicha categorización, no obstante, solo está fundamentada en la cantidad de población, y solamente genera efectos en términos de asignación de funciones. En Colombia, aunque la categorización territorial es una posibilidad, todavía no se ha desarrollado en los términos establecidos en la Constitución de 1991. Si se lograra serían los municipios los más beneficiados, y en particular lo serían los municipios más pequeños, los cuales se ven en la actualidad agobiados debido a que, aunque tienen asignada una importante cantidad de funciones sociales, en la práctica no pueden desarrollarlas de manera suficiente debido a la escasez de sus recursos propios. La uniformidad normativa que caracteriza a nuestras entidades territoriales impide que para muchas de ellas, en especial de nivel local, se pueda concretar la autonomía territorial. En la práctica su autonomía se manifiesta a niveles demasiado bajos, o en algunos casos es prácticamente inexistente.

Aunque Colombia ha acogido las diferentes alternativas de diseño institucional intergubernamental presentes también a nivel internacional, para garantizar la prestación directa, la categorización territorial sigue siendo un tema pendiente; sea que se implemente en los términos de la Constitución o a un nivel menos complejo, debe derivar en la diferenciación de los regímenes por categorías. Mientras esto no se haga, el diseño normativo que sustenta las entidades territoriales en nuestro país, en especial las del nivel local, seguirá siendo incoherente con sus debilidades y potencialidades y la fórmula del diseño intergubernamental seguirá estando incompleta. 


\section{REFERENCIAS}

Acto Legislativo 01 de 1945. Reformatorio de la Constitución Nacional. Congreso de la República de Colombia. Diario Oficial. Año Lxxx, n. ${ }^{\circ}$ 25769. 17 de febrero de 1945. P. 593.

Acto Legislativo 01 de 1968. Reformatorio de la Constitución Nacional. Congreso de la República de Colombia. Diario Oficial. Año. CV, n. ${ }^{\circ} 32673.17$ de diciembre de 1968. P. 641.

BIRD, R. (2001). Setting the Stage: Municipal and Intergovernmental Finance. En Freire, M. E. y Stern, R. E., eds. The Challenge of Urban Governance. Washington: World Bank Institute.

BoRJA, J. (1987). Dimensiones teóricas, problemas y perspectivas de la descentralización del Estado. ICI, Flacso y Clacso, Santiago de Chile.

Chile. Ley 18.695, Ley Orgánica Constitucional de Municipalidades (1998). Diario Oficial, 18 de agosto.

Chile. Subsecretaría de Desarrollo Regional-subdere (2009). Descentralización en América Latina: un análisis comparado de los casos de Colombia, Bolivia, Perú, Brasil y Argentina. Santiago de Chile: Subsecretaría de Desarrollo Regional y Administrativo.

Ciudades y Gobiernos Locales Unidos - CGLu. El acceso a los servicios básicos y el proceso de urbanización mundial. Barcelona: Tercer Informe Mundial sobre la Descentralización y la Democracia Local GOLD III. España. 2013. Disponible en: http://www.fomin. org/Portals/0/topics/uclg_resumenejecutivo.pdf

Ciudades y Gobiernos Locales Unidos - CGLu. El financiamiento de los gobiernos locales: los desafíos del siglo xxi. Segundo Informe Mundial sobre la Descentralización y la Democracia Local. Pamplona: Gold II, Resumen Ejecutivo, 2010. Disponible en: http:// www.uclg.org/sites/default/files/gold_ii_esp.pdf

Colombia (1991). Constitución Política. Bogotá: Presidencia de la República.

Colombia. Corte Constitucional. Sentencia C-004-93. Gaceta de la Corte Constitucional $1.1,1993,57$.

Colombia. Informe del Departamento Nacional de Planeación (2004). Balance del desempeño fiscal de los municipios y departamentos 2000-2003. Bogotá: Dirección de Desarrollo Territorial Sostenible DDTS.

Colombia. Informe del Departamento Nacional de Planeación (2006). Desempeño fiscal de los departamentos y municipios 2005 y comparativo 2004. Bogotá: Dirección Técnica de Desarrollo Territorial Sostenible-DDTs.

Colombia. Informe del Departamento Nacional de Planeación (2008). Desempeño fiscal de los departamentos y municipios 2007. Resultados de la gestión fiscal de los mandatarios locales 2006-2007. Bogotá: Dirección Técnica de Desarrollo Territorial Sostenible-DDTS.

Colombia. Informe del Departamento Nacional de Planeación (2010). Desempeño fiscal de los departamentos y municipios 2009. Bogotá: Dirección Técnica de Desarrollo Territorial Sostenible-DDTs. 
Colombia. Informe del Departamento Nacional de Planeación (2011). Desempeño fiscal de los departamentos y municipios 2010. Bogotá: Dirección Técnica de Desarrollo Territorial Sostenible-DDTS.

Colombia. Informe del Departamento Nacional de Planeación (2012). Desempeño físcal de los departamentos y municipios 2011. Bogotá: Dirección Técnica de Desarrollo Territorial Sostenible-DDTs.

Colombia. Informe del Departamento Nacional de Planeación (2013). Desempeño fiscal de los departamentos y municipios 2012. Bogotá: Dirección Técnica de Desarrollo Territorial Sostenible-DDTS.

Colombia. Informe del Departamento Nacional de Planeación (2014). Desempeño fiscal de los departamentos y municipios 2013. Bogotá: Dirección Técnica de Desarrollo Territorial Sostenible-DDTS.

Colombia. Ley 03 (1986). Por la cual se expiden normas sobre la administración departamental y se dictan otras disposiciones. Diario Oficial n. ${ }^{\circ} 37.304,10$ de enero.

Colombia. Ley 1176 (2007). Por la cual se desarrollan los artículos 365 y 357 de la Constitución Política y se dictan otras disposiciones. Diario Oficial n. ${ }^{\circ} 46.854,27$ de diciembre.

Colombia. Ley 136 (1994). Por la cual se dictan normas tendientes a modernizar la organización y el funcionamiento de los municipios. Diario Oficial n. ${ }^{\circ} 41.377,2$ de junio.

Colombia. Ley 1454 (2011). Por la cual se dictan normas orgánicas sobre ordenamiento territorial y se modifican otras disposiciones. Diario Oficial n. ${ }^{\circ} 48.115,29$ de junio.

Colombia. Ley 1551 (2012). Por la cual se dictan normas para modernizar la organización y el funcionamiento de los municipios. Diario Oficial n. ${ }^{\circ} 48.483,6$ de julio.

Colombia. Ley 60 (1993). Por la cual se dictan normas orgánicas sobre la distribución de competencias de conformidad con los artículos 151 y 288 de la Constitución Política y se distribuyen recursos según los artículos 356 y 357 de la Constitución Política y se dictan otras disposiciones. Diario Oficial n. ${ }^{\circ} 40.987,12$ de agosto.

Colombia. Ley 617 (2000). Por la cual se reforma parcialmente la Ley 136 de 1994, el Decreto Extraordinario 1222 de 1986, se adiciona la Ley Orgánica de Presupuesto, el Decreto 1421 de 1993, se dictan otras normas tendientes a fortalecer la descentralización y se dictan normas para la racionalización del gasto público nacional. Diario Oficial n. ${ }^{\circ} 44.188,9$ de octubre.

Colombia. Ley 715 (2001). Por la cual se dictan normas orgánicas en materia de recursos y competencias de conformidad con los artículos 151, 288, 356 y 357 (Acto Legislativo 01 de 2001) de la Constitución Política y se dictan otras disposiciones para organizar la prestación de los servicios de educación y salud, entre otros. Diario Oficial n. ${ }^{\circ}$ 44654, 21 de diciembre.

Colombia. Presidencia de la República (1993). Decreto 1421 de 1993. Por el cual se dicta el régimen especial para el Distrito Capital de Santafé de Bogotá. Diario Oficial n. ${ }^{\circ}$ 40.958. Bogotá, 21 de julio.

Contaduría General de la Nación. MinHacienda. www.contaduría.gov.co 
Cravacuore, D. (2007). Procesos políticos comparados en los municipios de Argentina y Chile: 1990-2005. Quilmes: Universidad Nacional de Quilmes.

DARío, I. et al. (2004). Crisis del centralismo y nuevos retos para las entidades territoriales: una mirada desde Colombia. Cuadernos del CENDES, n. ${ }^{\circ}$ 4, 23-54.

Díaz FlóRez, M. (2002). Federalismo fiscal y asignación de competencias: una perspectiva teórica. Economía Sociedad y Territorio.

Díaz, A. y Hernández, M. (2006). Relaciones intergubernamentales. Espacios Públicos. Vol. 9, n. ${ }^{\circ} 18,36-53$.

Duque, N. (2009). La autonomía territorial en el marco de la república unitaria y la capacidad institucional del Estado subnacional en Colombia. Administración \& Desarrollo, Escuela Superior de Administración Pública. Vol. 37, n. ${ }^{\circ}$ 51, 109-124.

Duque, N. (2010). El departamento en las relaciones intergubernamentales. Opera. n. ${ }^{\circ}$ $10,233-246$.

Duque, N. (2009). La autonomía territorial en el marco de la república unitaria y la capacidad institucional del Estado subnacional en Colombia. Administración \& Desarrollo, Escuela Superior de Administración Pública, vol. 37, n. ${ }^{\circ}$ 51, 109-124.

Duque, N. (2012). Particularidades de la Ley Organica de Ordenamiento Territorial. Análisis Político, vol. 25, n. ${ }^{\circ}$ 76, 175-190.

Eguino, H. et al. (2010). Estudio de las características estructurales del sector municipal en América Latina. Documento de Debate IDB-DP-145. Washington D.C.: Inter-American Development Bank.

Finot, I. (2007). Los procesos de descentralización en América Latina/The Processes of Decentralization in Latin America. Investigaciones Regionales, . $^{\circ} 10$.

Gaitán, P. y Ospina, C. M. (1992). Poder local: realidad y utopía de la descentralización en Colombia. Bogotá: Instituto de Estudios Políticos y Relaciones Internacionales.

Gómez-Pomar, J. y Sanagustín, M. G. (2010). Por un Estado autonómico racional y viable. Fundación para el Análisis y los Estudios Sociales-FAES.

GonZÁlez CRUZ, F. (2000). El régimen municipal en la Constitución venezolana de 1999. Revista Iberoamericana de Administración, n. ${ }^{\circ} 5$.

Huerta Moreno, M. G. (2005). El neoliberalismo y la conformación del Estado subsidiario. Política y Cultura, n. $^{\circ} 24,121-150$.

Iturburu, M. S. (2000). Potestades y restricciones constitucionales para un nuevo modelo de gestión local. Buenos Aires: Instituto Nacional de la Administración Pública (INAP).

Massiris-Cabeza, A., et al. (2012). Procesos de ordenamiento en América Latina y Colombia. Bogotá: Universidad Nacional de Colombia.

Moncayo Jiménez, E. (2006). La transformación del Estado en América Latina: una perspectiva económica desde los países andinos. Problemas del Desarrollo. Vol. 37, n. ${ }^{\circ}$ $147,135-159$. 
Oszlak, O. (2003). El mito del Estado mínimo: una década de reforma estatal en la Argentina. Desarrollo Económico. Vol. 42, n. ${ }^{\circ} 168$, enero-marzo, 519-543.

Pinilla, D. E.; De Dios Jiménez, J. y Montero, R. (2015). La descentralización fiscal en América Latina. Balance de un proceso. Revista de Economía Institucional, Vol. 17, n. ${ }^{\circ} 33,133-160$.

Portp, A. (2008). El funcionamiento del federalismo fiscal argentino. Nota sobre el financiamiento de las municipalidades y la "tasa de abasto". Documento de Trabajo n. ${ }^{\circ} 71$. Universidad Nacional de la Plata, Departamento de Economía.

Quenan, C.; Velut, S. y Jourcin, E. (2014). Los desafíos del desarrollo en América Latina. Dinámicas socioeconómicas y políticas públicas. Institut des Amériques, Col. A Savoir.

RAmírez, E. L. (2015). Las paradojas de las políticas centralistas en relación con la autonomía de las entidades territoriales en Colombia. Revista Derecho del Estado, n. ${ }^{\circ} 35,41-63$.

Venezuela. Gobierno Bolivariano de Venezuela. Constitución de la República Bolivariana de Venezuela. Gaceta Oficial, 1999, n. ${ }^{\circ} 5.453$.

Venezuela. Ley Orgánica del Poder Público Municipal (2006). Gaceta Oficial n. o 5.806, 10 de abril.

Venezuela. Reforma parcial de la Ley Orgánica de Descentralización, Delimitación y Transferencia de Competencias del Poder Público (2009). Gaceta Oficial de la República Bolivariana de Venezuela. ‥ ${ }^{\circ} 39.140,17$ de marzo de 2009.

WIESNER, E. et al. (2002). Transferencias, incentivos y la endogenidad del gasto territorial. Bogotá: Departamento Nacional de Planeación.

ZAPATA, J. G. (2010). Las finanzas territoriales en Colombia. Bogotá: Fedesarrollo y CAF. 